Efectividad de la inversión pública peruana en los logros educativos.

Peruvian Public Investment Effectiveness in educational achievements.

Línea de Investigación: Políticas Públicas

Recibido: 11 de marzo de 2021

Aprobado: 16 de mayo de 2021

\section{AUTOR 1:}

Ronald Nilton Silva Gil

Ingeniero Economista, MBA, Magister en

Gerencia Pública, Universidad Nacional de Ingeniería, Lima, Perú. Consultor inversiones.

ORCID:

https://orcid.org/0000$\underline{0001-7221-6108}$

Correo: rsilvagil@gmail.com

\section{AUTOR 2:}

Nora Gina del Pilar Tejada Vidal

Economista, MBA. Doctora. Universidad de Lima, Perú. Universidad Autónoma de Madrid: Madrid, Madrid, España. Labora en la Universidad Científica del Sur, Lima, Perú.

ORCID:

https://orcid.org/00000002-8498-9826

Correo: ptejada.sis@gmail.com

\section{RESUMEN:}

Perú presenta logros educativos desiguales, pero algunos departamentos destacan positivamente. La investigación explica si la inversión pública en educación es significativa para analizar los logros educativos, determinando si la comprensión lectora se explica por la inversión ejecutada en educación en el período 2007-2016. La base para ello es la teoría de la producción educativa, que señala que los logros educativos están determinados por los años de escolaridad, las características de la escuela, el perfil del maestro, las capacidades del niño y las características y contribuciones de los hogares. Como resultado, se demuestra que la inversión pública ejecutada en educación no es una variable significativa para explicar los cambios en los logros educativos en el Perú. Sin embargo, se demostró que los logros educativos se explican por variables que no se analizan comúnmente como variables educativas, como son por ejemplo la desnutrición infantil, la cobertura de agua, electricidad o internet. En conclusión, significa que la inversión pública en educación en el Perú debería priorizar también sus intervenciones en este tipo de variables. Es decir, complementar las tradicionales intervenciones en escuelas y los maestros, con variables referidas a las condiciones de los estudiantes y las condiciones para el estudio que cuentan los hogares.

Palabras clave: Inversión, Eficiencia de la educación, Rendimiento escolar, Economía, Economía de la educación.

\section{ABSTRACT:}

Peru presents uneven educational achievements, but some departments stand out positively. The research explains whether public investment in education is significant for analyzing educational achievements, determining whether reading comprehension is explained by the investment made in education in the 2007-2016 period. The basis for this is the theory of educational production, which indicates that educational achievements are determined by the years of schooling, the characteristics of the school, the profile of the teacher, the capacities of the child, and the characteristics and contributions of households. As a result, it is shown that public investment in education is not a significant variable to explain changes in educational achievements in Peru. However, it was shown that educational achievements are explained by variables that are not commonly analyzed as educational variables, such as child malnutrition, clean water, electricity or internet coverage. In conclusion, it means that public investment in education in Peru should also prioritize its interventions in these types of variables. That is, to complement the traditional interventions in schools and teachers, with variables referring to the conditions of the students and the conditions for the study that the homes have.

Keywords: Investment, Educational efficiency, Academic achievement, Economics, Economics education. 


\section{INTRODUCCIÓN}

El tema de investigación está referido a, si la inversión pública en educación es significativa para explicar los logros educativos del Perú. Se justifica en la necesidad de conocer si la influencia de la inversión pública en los logros educativos permitirá a los tomadores de decisiones de política, establecer los niveles de esfuerzo de gasto público que resulten necesarios realizar sobre cada una de las variables a fin de mejorar los logros educativos en la formación de los niños. Además, permitirá contar con instrumentos de medición para evaluar las políticas públicas en educación. El objetivo es determinar si la comprensión lectora se explica por la inversión pública ejecutada en educación en el período 2007-2016. Con ello, se busca establecer la contribución relativa de la inversión pública en los logros educativos. Para ello, la investigación utiliza las bases teóricas de la función de producción de educación, que señala que los logros educativos están determinados por los años de escolaridad, el conjunto de características de la escuela y el maestro, las características del niño, las características del hogar y los aportes educativos que contribuyen los hogares. En la presente investigación se utilizan variables proxy de estos elementos, teniendo que logros educativos están determinados por la disponibilidad de locales escolares con aulas en buen estado, inversión pública por alumno acumulado en el periodo, gasto corriente por alumno, desnutrición crónica, cobertura en el abastecimiento de agua, cobertura en el alumbrado eléctrico y la cobertura en el acceso a internet.

De acuerdo a la metodología tipo causal o correlacional utilizada, se busca establecer la relación causa efecto entre los logros educativos y las variables anteriormente indicadas. Para ello, se aplica un modelo econométrico de panel data para realizar un análisis comparativo de la evolución de estas variables para los diferentes departamentos del Perú. Las hipótesis, plantean que la inversión pública en educación en el periodo 2007-2016 no explica significativamente los logros de aprendizaje de los alumnos de educación primaria en el Perú. Por el contrario, existen otras variables a partir de la función de producción que explican los logros educativos, como son la desnutrición 
crónica, el abastecimiento de agua en los hogares, el acceso a alumbrado eléctrico o el acceso a Internet en los hogares.

\section{MARCO TEORICO}

La presente investigación se basa en la Economía de la Educación. Al respecto, se señala que:

La economía de la educación es una ciencia social aplicada, que investiga y estudia la dimensión económica y las posibles alternativas conducentes para que los recursos materiales y financieros limitados produzcan y satisfagan la necesidad y el derecho que tienen a la educación los individuos, los grupos y las instituciones. (Fermoso, 1997)

(Lassibille, 2012) señala que la economía de la educación se inicia con la teoría del capital humano, donde destacan los aportes de Fisher, Schultz, el premio nobel Gary Becker, Arrow, Spencer, entre otros. Luego, se analizan otros temas, como son los efectos no monetarios de las inversiones educativas, el fenómeno de la sobre educación, la relación de la educación con el crecimiento económico, la producción de servicios educativos, sus costos y financiación, entre otros.

La investigación se basa específicamente en la temática de la producción de servicios educativos, donde destacan los aportes de Coleman, que equipara al sector educación como una industria, donde el centro educativo es una empresa multiproducto, a la cual se puede aplicar la teoría de la producción. Este enfoque contribuye a crear un uso más racionalizado de los recursos que se entregan a los sistemas de enseñanza y contar con medios de evaluación de las políticas educativas. Ello generó la estandarización de instrumentos de medición, que han derivado en pruebas como PISA u otros mecanismos de evaluación (Lassibille, 2012).

Respecto a la temática de inversión pública en educación, se señala que los países han incrementado la inversión pública en educación por sus demostrados efectos positivos, económicos y sociales. Sin embargo, en términos de política pública, es necesario entender cómo impacta la inversión pública en la calidad educativa, a fin de 
contar con mejores mecanismos para priorizar las inversiones y hacerlas más eficientes (Bayona Rodriguez, 2016). Así, en la literatura existente, se observa que se indagan sobre los efectos de la infraestructura en la calidad educativa. De estos estudios, se concluye que los colegios con mejor infraestructura reducen las tasas de repetición de sus estudiantes. Este resultado es coherente con la evidencia encontrada en la literatura, que señala que mejores ambientes escolares generan mejores procesos educativos. Es interesante esta conclusión pues señala que, la infraestructura diferenciada como los laboratorios, en sus distintos tipos, genera un impacto incremental sobre la calidad educativa (Bayona Rodriguez, 2016). De otro lado, se identifica otra línea de investigación, la cual persiste en encontrar la relación existente entre el estado de la infraestructura de las escuelas de educación básica para América Latina y las relaciones con los resultados de los alumnos en las pruebas de lenguaje y matemáticas en tercer y sexto grado de educación básica. Este estudio parte del hecho que existe en la literatura de otros países evidencia sobre las asociaciones positivas entre las condiciones físicas de las escuelas y sus resultados académicos. Se observa que los factores que están más altos y significativamente asociados con los aprendizajes son la presencia de espacios de apoyo a la docencia, (bibliotecas, laboratorios de ciencias y salas de cómputo), la conexión a servicios públicos de electricidad y telefonía y la existencia de agua potable, desagüe y baños en número adecuado. En las escuelas urbanas además de los anteriores, la presencia de áreas de uso múltiple (gimnasio y/o auditorio) y de espacios para enfermería o servicios psicopedagógicos están asociados con mejores aprendizajes de los estudiantes (Duarte \& Carlos \& Moreno, 2011). En la misma línea, en otra investigación se encuentra que aun cuando la inversión en textos escolares destaca como sustancialmente más positiva, se sigue priorizando la inversión en infraestructura (Coraggio, 1995).

De otro lado (UNESCO, 2017) menciona la implementación de otros modelos educativos, como los Colegios de Alto Rendimiento-COAR, para mejorar los logros educativos. Estos colegios destacan por la puesta en marcha de un servicio totalmente 
diferenciado del modelo de oferta educativa existente. Para esta política en Perú, se prevé invertir 960 millones de soles hasta el 2025 para implementar un colegio de alto rendimiento, COAR en cada una de las regiones.

En la misma temática de la inversión pública, (Palacios, 2018) indica que en el periodo de estudio del 2000 al 2015 en el Perú, la inversión y el gasto en educación presentan una tendencia creciente. Pero también se ha incrementado la infraestructura educativa en mal estado. Lamentablemente, se ha profundizado la brecha en la infraestructura educativa en dicho periodo. Esta situación estaría contribuyendo negativamente con la calidad educativa. Sin embargo, estas inversiones podrían estar demostrando que, a pesar del incremento en infraestructura, no resulta suficiente para revertir y hacer retroceder las brechas en infraestructura educativa. Esto lleva a plantear que los esfuerzos de inversión han sido insuficientes para cerrar las brechas de infraestructura. A pesar de haber sido crecientes, la inversión tiene un efecto positivo, pero requieren de mayores esfuerzos (Arce Vega \& Tejada Vidal, 2017). Ciertamente, la infraestructura per se no resulta relevante sino su relación con la efectividad en los logros educativos. En ese sentido, si la infraestructura tuviera un efecto preponderante en los logros educativos, esta debería priorizarse. Por otro lado, si hubiera otros factores más importantes, quizá los recursos deban orientarse a dichas variables que significan mayores efectos en los logros educativos y las inversiones en infraestructura, deberían ser más complementarios. (Allanta, 2017) afirma que la infraestructura educativa mejoró la calidad educativa y amplió las oportunidades de aprendizaje de los estudiantes. Agrega también que se evidencia una correlación entre el desarrollo cognitivo y el ambiente en donde los alumnos realizan sus actividades pedagógicas. Así, define algunas características que mejoran como los alumnos perciben los conocimientos cuales son, aulas con buena ventilación e iluminación, áreas verdes de esparcimiento. En ese sentido, recomienda que las futuras edificaciones educativas evalúen el cumplimiento de estas consideraciones antes de su implementación. Finalmente, recomienda que estos nuevos diseños de áreas complementarias para el aprendizaje 
no responden a las normas actuales. En ese sentido, precisa Allanta, como necesario realizar una mejor evaluación al momento de diseñar infraestructura educativa que responda a los objetivos de aprendizaje de las zonas en donde se edifican.

Por otro lado (MINEDU, 2016), específicamente referido a infraestructura escolar en relación a los profesores, señala que en general se carece de espacios y servicios diseñados en función a las necesidades de estos, lo cual no facilita su trabajo y la interacción con padres de familia, colegas o directores. Esta infraestructura está referida a salas de profesores, servicios higiénicos exclusivos para ellos, espacios de preparación de clases o descanso, entre otros. Agrega que se han dictado lineamientos de infraestructura orientada al docente, los cual servirá para construir nuevas infraestructuras y adecuar las existentes. En el caso de educación en Finlandia, existen explicaciones que relevan el rol del docente, pero sólo le facilitan un ambiente de trabajo profesional (Scott, 2012) no un espacio para relajarse y generar ideas de manera más tranquila y creativa. En ese sentido, el ambiente para docentes debe comprender aspectos de condiciones organizativas y de bienestar, que incluya las condiciones físicas. Para el caso de Finlandia, se indica que las escuelas son bien diseñadas y equipadas, con emplazamientos adecuadamente mantenidos, que hacen de ellos lugares estimulantes para el trabajo.

Sin embargo, en la temática de los logros educativos, se pueden identificar otro tipo de estudios relacionados a la gestión educativa. Entre ellos, se destaca un estudio (UNESCO, 2017), donde se señala que un ejemplo de una estrategia diferenciada que ha sido implementada en los últimos años es la extensión de la Jornada Escolar Completa (JEC) a nivel secundario, para lo cual se han incrementado las horas lectivas. Como otro aspecto relacionado a los logros educativos, se plantea en este estudio que se debe considerar la marcada heterogeneidad de los gobiernos locales que exige $u$ obliga a que estos reciban un tratamiento diferenciado para definir su papel en la educación (UNESCO, 2017). 
De otro lado a nivel de Perú, (Mamani, 2018) identifica como variables que explican los resultados de la Evaluación Censal Escolar (ECE), a la calidad educativa, clima escolar, participación de los padres de familia, factor personal de los estudiantes. En cuanto a la percepción de la Calidad Educativa, la explica por la formación docente y el fortalecimiento del liderazgo educativo, la gestión de servicios educativos, los factores asociados a la calidad y condiciones básicas de las instituciones educativas para el aprendizaje y el fortalecimiento de las competencias básicas. La percepción de clima escolar lo aborda con la organización de aula, convivencia entre estudiantes y profesores, y la relación entre profesores. (Mamani, 2018) concluye que la calidad educativa, el clima escolar, la participación de los padres de familia y los aspectos personales de los estudiantes en el caso de estudio en Tacna, influyeron sobre el logro en lectura en la ECE 2016. (Rodríguez Mendoza, 2018) destaca que el trato del profesor influye en clima escolar, que puede afectar el aprendizaje. Sin embargo, no se muestra la ponderación relativa de estos factores, haciéndose difícil determinar cuál de los factores influyó más en la evaluación.

La función de producción en educación, La producción de la educación es parte de la economía de la educación que busca comprender cómo las instituciones educativas combinan ciertas insumos o entradas, como son los docentes, libros, computadoras, con el fin de producir un bien o servicio educativo. Estos bienes o servicios finales pueden medirse como las notas de exámenes, éxito escolar o la matrícula de estudiantes. Para su estudio se utiliza la función de producción, que intentan mostrar los volúmenes de bienes educativos o salidas producidas desde determinadas cantidades o conjunto de entradas (Carnoy, Miller, \& Luschei, 2006).

(Morduchowics, 2004) define a la función de producción como una relación matemática que explica como los recursos pueden ser convertidos en productos. Asimismo, (MINEDU, 2016) señala que los factores que determinan los logros del aprendizaje son la calidad de la infraestructura educativa, la gestión escolar, el diseño curricular, la dotación de materiales educativos, las características de los estudiantes 
(habilidades, actitudes, su origen económico y familiar), así como el desempeño docente, el cual se considera como el más importante. Estos aspectos determinan las condiciones en las que estudian los alumnos y enseñan los profesores.

(Carnoy, 2013) afirma que a partir de los resultados de las modelaciones de las funciones de producción se pueden evaluar y proponer a los gobiernos políticas educativas a ser implementadas. Además, (Carnoy, 2013) afirma que para poder determinar la aplicación de la teoría de la producción clásica a la producción de la educación se debe evaluar su pertinencia a la lógica educativa. En la teoría de la producción la empresa busca maximizar el beneficio. Por tanto, se debe analizan las variables sobre un resultado específico en educación, a fin de verificar si los supuestos se cumplen. Es decir, si estamos analizando los logros educativos, debemos suponer que el objetivo de la escuela no es maximizar su beneficio sino maximizar los logros educativos.

La teoría adoptada por los autores en este artículo sobre función de producción en educación corresponde a la propuesta por (Glewwe, Lambert, \& Chen, 2020). Este autor analiza la educación como un proceso productivo que puede resultar extraño, pero reflexionar sobre ello, puede resultar un enfoque útil para proveer un marco donde sea posible comprender como las habilidades cognitivas (lectura y matemáticas) y no cognitivas (habilidades sociales y organizacionales) son generadas a través de la educación formal y tener una guía para conocer cómo utilizar la data de educación para estimar el impacto de las políticas sobre la adquisición de habilidades en los estudiantes. En ese sentido, plantea una ecuación para describir ello:

$$
A=\boldsymbol{a}(S, Q, C, H, I)
$$

Donde:

- A : habilidad aprendida (logro).

- $\quad$ S : años de estudio.

- Q : set de características del colegio y los profesores.

- C : características del niño (incluye sus habilidades) 
- H : características del hogar que afectan el aprendizaje

- I : insumos para la educación que el hogar contribuye (asistencia, compra de textos y otros suplementos para la escuela)

Si bien es cierto los años de estudio (S) y los insumos para la educación que en las que el hogar contribuye (I) son variables que pueden ser agrupadas con las variables del niño o del hogar, se separan de $\mathrm{C}$ y $\mathrm{H}$ por qué casi todas estas siempre están bajo el control de los padres. Esta base teórica será utilizada para el análisis de las variables que afectan los logros educativos, tema a analizar en la presente investigación.

En cuanto a la identificación del tema y estructura de la investigación, el problema de investigación cuestiona si la inversión pública ejecutada en el periodo 2007-2016 en el Perú se relaciona con los logros educativos alcanzados por los diferentes departamentos. Para ello, se ha identificado un marco teórico específico referido a la función de producción educativa, que explica las variables referidas a los logros educativos. En la presente investigación se partirá de dicho marco teórico, analizando para el caso peruano las variables del modelo. Luego, se plantea un modelo econométrico que relaciona las variables analizadas, que explique la relación existente entre ellas.

\section{METODOLOGÍA}

La investigación es de tipo causal o correlacional. El tipo de estudio corresponde a una investigación aplicada, de diseño descriptivo y no experimental. En cuanto al período de estudio, es longitudinal y transversal, es decir mixto. La variable dependiente de la investigación es la Comprensión Lectora: Alumnos que logran los aprendizajes del grado (\% de alumnos de $2^{\circ}$ grado participantes en evaluación censal). Las variables independientes consideradas son la disponibilidad de locales escolares con aulas en buen estado, gasto de capital per cápita acumulado, gasto corriente per cápita, desnutrición crónica, cobertura en el abastecimiento de agua, cobertura en el alumbrado eléctrico y la cobertura en el acceso a internet. Con esta información cuantitativa, se busca la prueba de la hipótesis y la interpretación de los resultados encontrados. 
(ESCALE-MINEDU, 2020) muestra que en el año 2016 el departamento de Tacna obtuvo el mayor porcentaje, cercano al $80 \%$, de alumnos que logran los aprendizajes en Comprensión de Textos. En contraste, en el mismo año el departamento de Loreto obtuvo el resultado más bajo, cercano al $20 \%$. Lo anterior conlleva a preguntarse sobre cuáles son las razones que explican las diferencias tan marcadas en Comprensión de Textos entre los departamentos en el Perú (Ver Figura 1).

Figura 1: Comprensión de Textos: Alumnos que logran los aprendizajes del grado (\% de alumnos de $2^{\circ}$ grado participantes en evaluación censal). Comparativo 2007 Vs. 2016

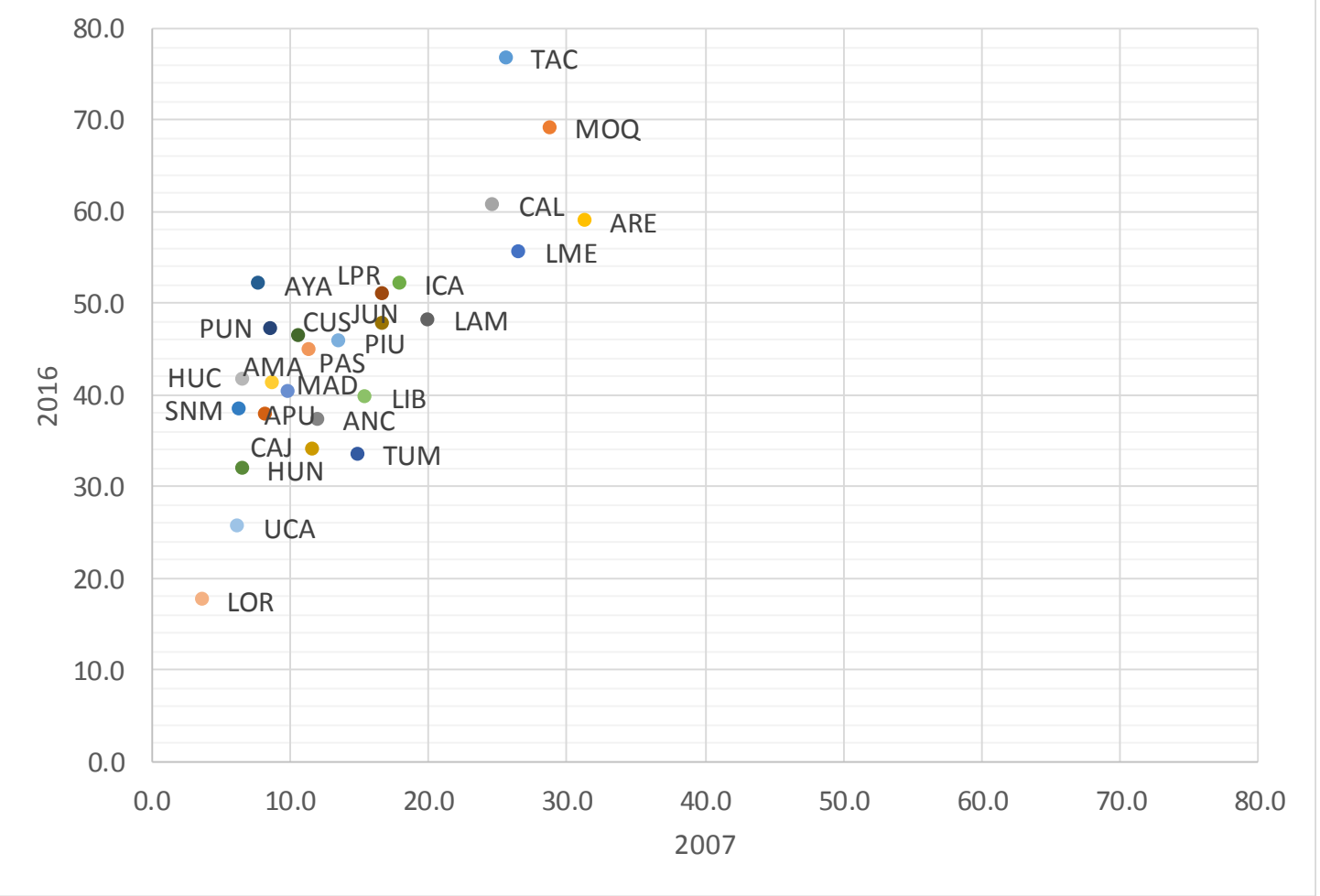

Fuente: (ESCALE-MINEDU, 2020)

Para explicar estas diferencias en comprensión lectora se puede usar la función de producción de educación. Adaptando el modelo de (Glewwe, Lambert, \& Chen, 2020) a las variables a analizar, se tiene la siguiente ecuación:

$$
A=a(S, \boldsymbol{Q}, \boldsymbol{C}, \boldsymbol{H}, \boldsymbol{I})
$$

Sin embargo, las variables anteriores requieren para su análisis contar con una definición operativa, que permita estudiar comparativamente su comportamiento en el tiempo. Para ello, se ha planteado un conjunto de variables a analizar (Ver Tabla 1). 
Tabla 1: Identificación de variables operativas del modelo adaptado de la función de producción de educación

\begin{tabular}{ccc}
\hline$N^{\circ} \quad$ Variable del Modelo & Variable operativa para análisis \\
\hline & - & Comprensión Lectora: Alumnos que \\
& logran los aprendizajes del grado \\
& (\% de alumnos de 20 grado \\
& participantes en evaluación censal). \\
& Corresponde al Porcentaje de \\
& alumnos de un nivel y grado \\
& determinado que alcanzaron los \\
& objetivos de aprendizaje esperados \\
& en la competencia Comprensión de \\
& Textos, de acuerdo a la estructura \\
& curricular vigente.
\end{tabular}

Q: Conjunto de características del colegio y los profesores
- Porcentaje de locales escolares con aulas en buen estado.

- Gasto corriente percápita por alumno matriculado ejecutado por el Estado.

- Gasto de capital percápita acumulado por alumno matriculado ejecutado por el Estado.

- Desnutrición Crónica

C: Conjunto de características del niño

$\mathrm{H}$ : Conjunto de características del hogar que afectan el aprendizaje

I: Insumos para la

6 educación que el hogar otorga.

Fuente: Elaboración propia. Adaptado de (Glewwe, Lambert, \& Chen, 2020).

- Porcentaje de Hogares en viviendas particulares que disponen de alumbrado eléctrico por Red Pública.

- Porcentaje de Hogares que acceden al servicio de internet.

\section{Supuestos}

Se considera como la variable que medirá el logro educativo de la prestación del servicio educativo.

Variable considerada como constante (Ceteris Paribus) para el modelo.

Se analiza las variables referidas únicamente a las características del colegio o institución educativa.

Las variables referidas a las características de los profesores son considerada constante (Ceteris Paribus) para el modelo.

Se considera a la desnutrición crónica como una variable referida a una condición básica de salud para poder estudiar.

Asimismo, el acceso al agua como variable que afectaría la salud del niño.

Se considera que el alumbrado y el acceso a internet en el hogar representan variables sobre las condiciones del hogar que afecta el aprendizaje.

Variable considerada como constante (Ceteris Paribus) para el modelo.

A continuación, se describe estadísticas de estas variables. En el año 2019, Tacna

destaca como la región que tiene cerca del $70 \%$ de sus locales escolares en buen

Estado. Y el contraste es con el caso de Loreto, que presenta un poco más del $10 \%$ de sus locales escolares en buen estado. Para el caso de esta variable, se puede observar que los departamentos que tienen mayor logro educativos son también los que presentan un mayor porcentaje de locales escolares en buen estado (Ver Figura 2). ISSN: 2414-4991. Creative Commons Atribución-NoComercial-Compartirlgual 4.0 Internacional. (c)(1)() 
Figura 2: Locales escolares con aulas en buen Estado

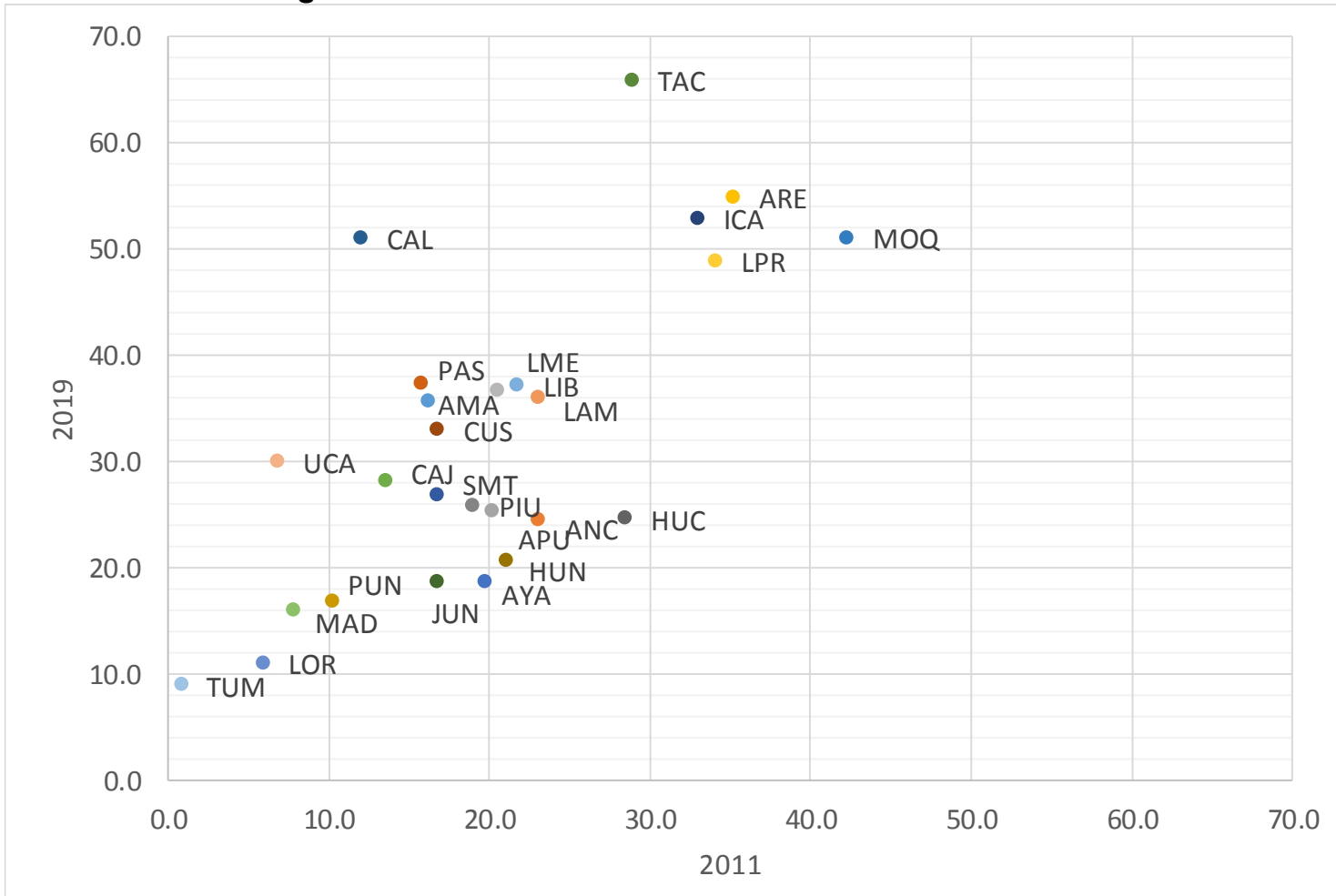

Fuente: (INEI, Instituto Nacional de Estadística e Informática-INEI, 2020)

En cuanto al Gasto Corriente y Gasto de Capital Per cápita, se analizó el gasto promedio anual por alumno matriculado en el periodo 2009 al 2018 (Ver Figura 3). Al respecto, se tiene que Moquegua destaca con el mayor monto, destinando aproximadamente más de 1,200 soles en gasto de capital y más de 2,500 soles en gasto corriente. Sin embargo, Loreto se sitúa por debajo de los 400 soles por alumno en gasto de capital y aproximadamente en 1,500 soles en gasto corriente. 
Mg. Ronald Nilton Silva Gil. ORCID: 0000-0001-7221-6108 Dra. Nora Gina del Pilar Tejada Vidal. ORCID: 0000-0002-8498-9826

Figura 3: Gasto Corriente y Gasto de Capital percápita por alumno matriculado en Educación Básica Regular por departamento en el periodo 2009-2018 (S/)

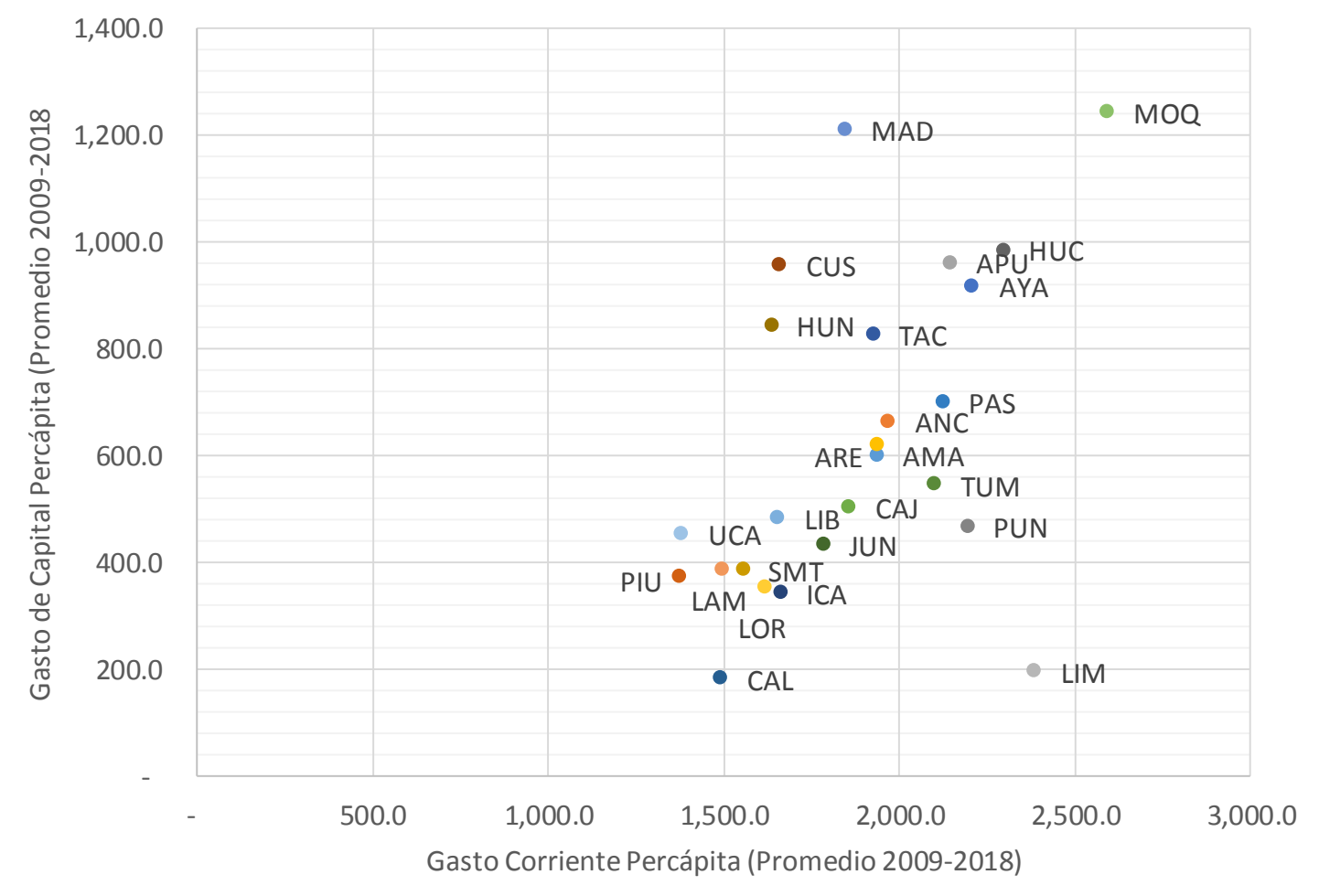

Fuente: Elaboración propia con información (INEI, Instituto Nacional de Estadística e InformáticaINEI, 2020) (Consulta Amigable MEF, 2020)

En cuanto a la desnutrición crónica, se observa que las regiones que presentan el menor porcentaje coinciden con aquellas regiones que tienen los mayores logros educativos en comprensión lectora. Y por el contrario, por ejemplo Loreto, Ucayali y Huánuco, que presentan menores logros educativos en comprensión lectora, estos coinciden con sus mayores niveles relativos en desnutrición crónica (Ver Figura 4). 
Figura 4: Desnutrición Crónica Infantil (DCI)1, comparativo 2009 Vs. 2018 por departamentos (Perú)

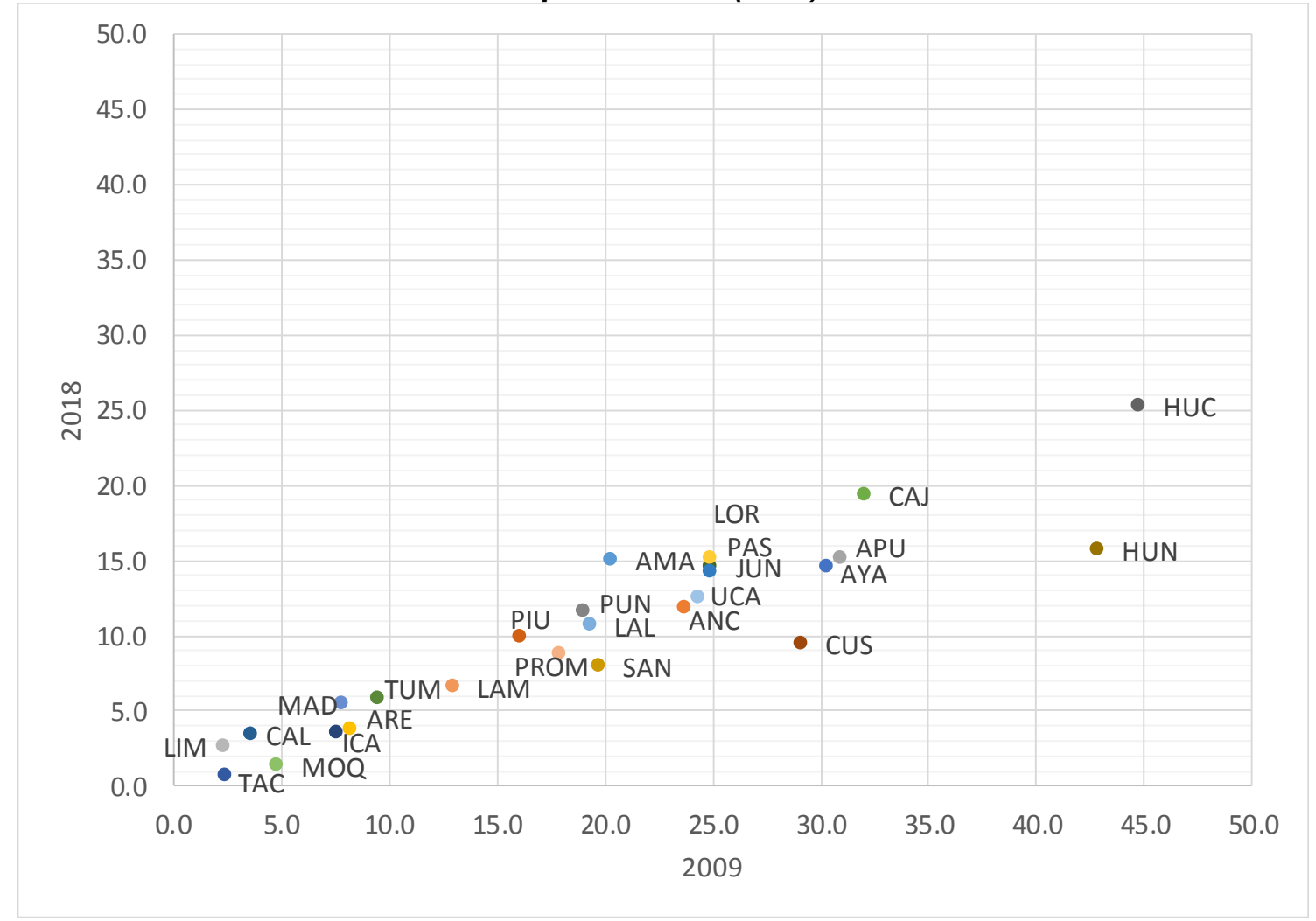

Fuente: Elaboración propia con información (MINSA, 2020)

En cuanto al abastecimiento de agua, destacan con coberturas de cercanas al $95 \%$ los departamentos de Lima, Callao, Moquegua, Tacna y Arequipa. En contraste, de nuevo figura Loreto, que presenta la menor cobertura, con cerca del $55 \%$. Se puede observar un común denominador. Aquellas regiones con mayor cobertura de agua, también son las que tienen mayores logros y viceversa (Ver figura 5)

\footnotetext{
${ }^{1}$ La información se reporta como la proporción de menores de 5 años con desnutrición crónica. De acuerdo al "Plan Nacional para la Reducción y Control de la Anemia Materno Infantil y la Desnutrición Crónica Infantil en el Perú: 2017-2021", la Desnutrición Crónica Infantil (DCI) es el estado por el cual una niña o un niño presentan retardo en su crecimiento de talla para su edad.
} 
Mg. Ronald Nilton Silva Gil. ORCID: 0000-0001-7221-6108 Dra. Nora Gina del Pilar Tejada Vidal. ORCID: 0000-0002-8498-9826

Figura 5: Hogares que se abastecen de agua mediante red pública (Porcentaje), comparativo 2007 Vs. 2016

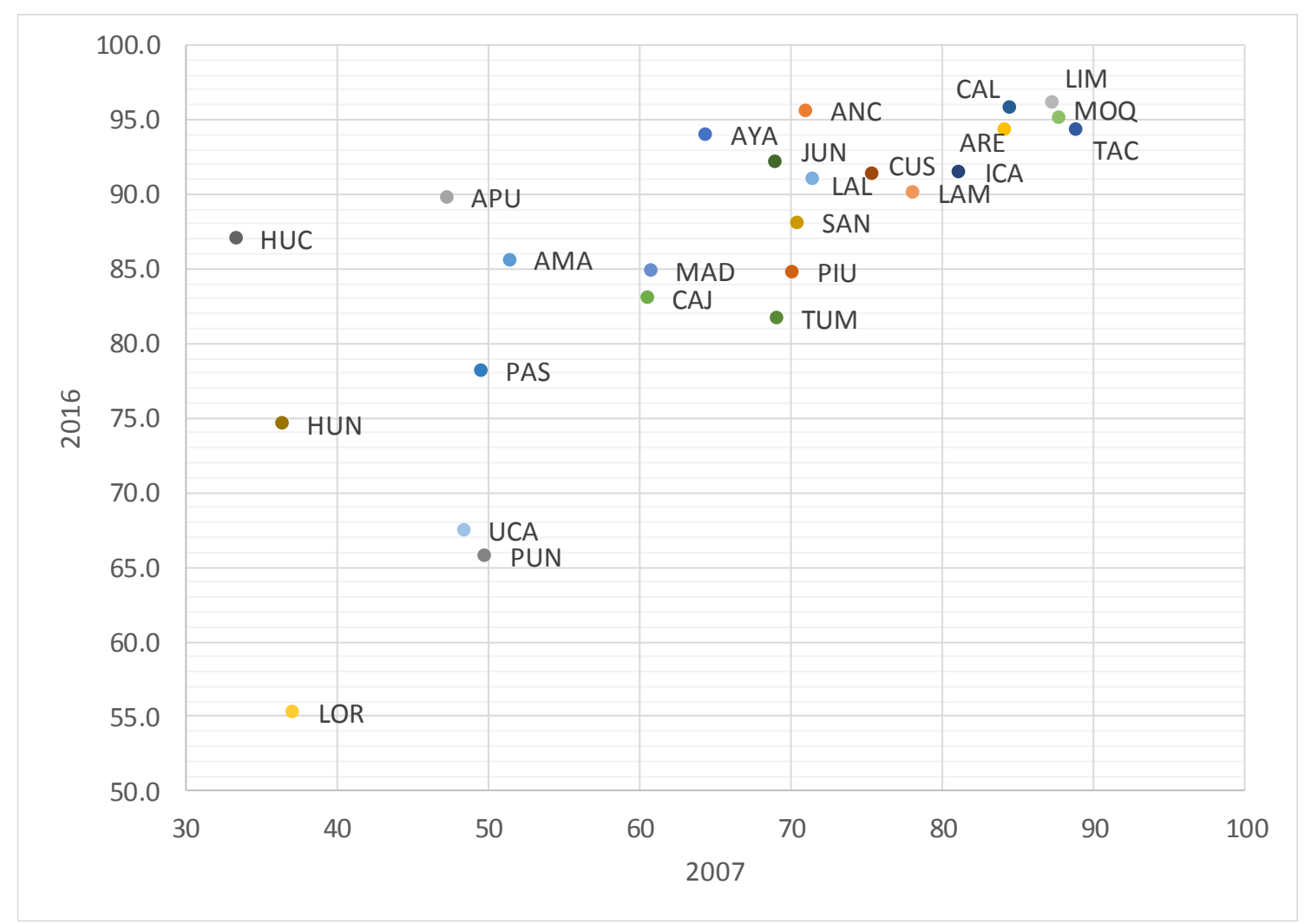

Fuente: Elaboración propia con información de (INEI, Sistema de Información Regional para la toma de decisiones, 2020)

En cuanto al alumbrado eléctrico, los departamentos que más han avanzado en cobertura son Lima, Callao, Tumbes, Ica, Arequipa, que están sobre el $97 \%$ de cobertura. En el otro extremo se encuentra Loreto y Amazonas, que tienen menos del $85 \%$ de cobertura. De manera reiterativa, los departamentos con mayor cobertura de alumbrado eléctrico también coinciden con aquellas que han experimentado mayores logros educativos (Ver Figura 6). 
Figura 6: Hogares en viviendas particulares que disponen de alumbrado eléctrico por Red Pública, comparativo 2007 Vs. 2018 por departamentos (Perú)

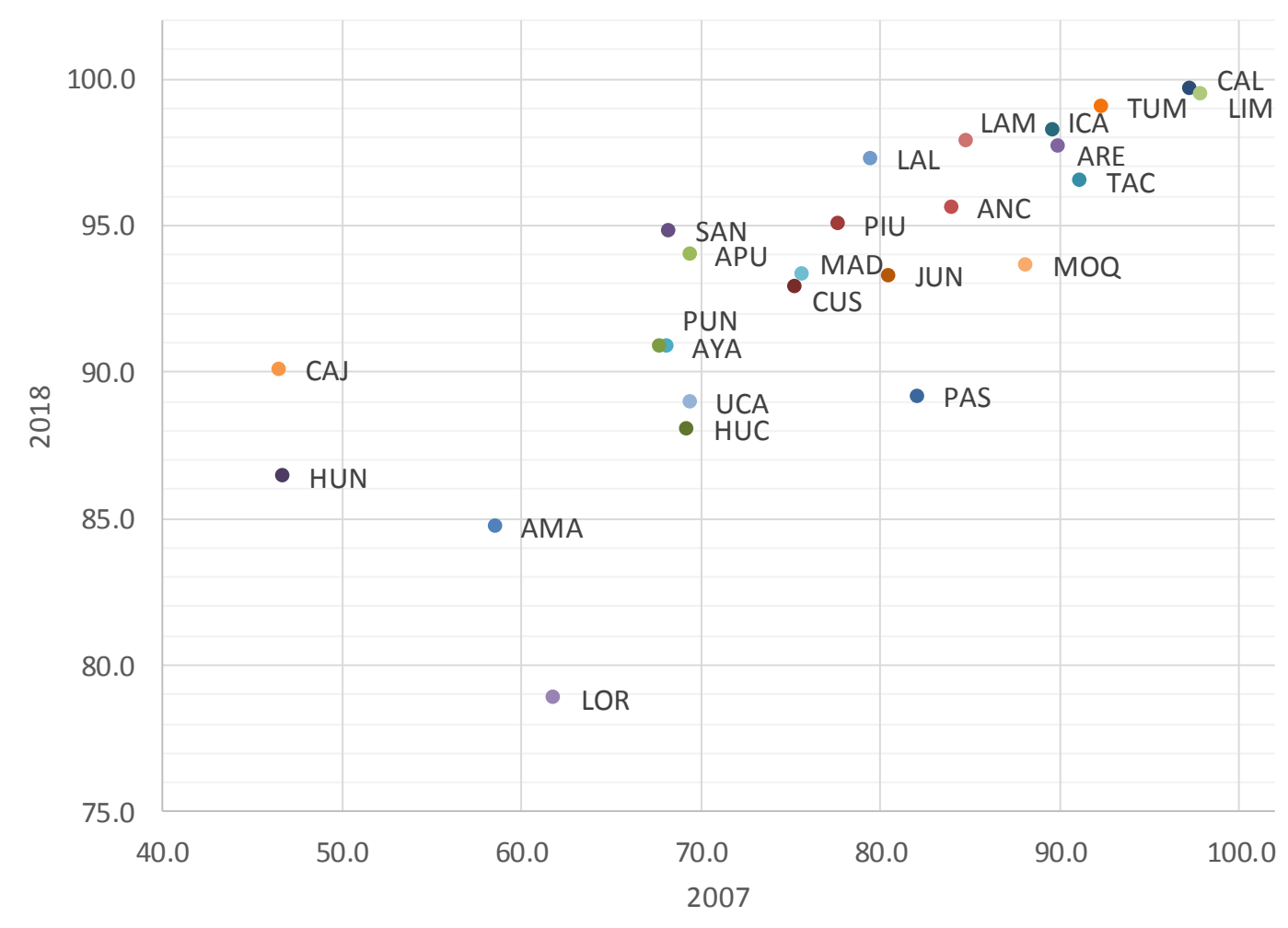

Fuente: Elaboración propia con información de (INEI, Instituto Nacional de Estadística e Informática-INEI, 2020)

El acceso a Internet en departamentos como Lima, Callao y Tacna está sobre el $40 \%$. Otro grupo de departamentos como Arequipa, Ica y Moquegua superan el 30\%. Pero hay un grupo importante de departamentos como Huancavelica, Apurímac, Ayacucho, Pasco, Huánuco y Cajamarca, que no superan el 10\% de cobertura. Loreto está cercano al $20 \%$ de cobertura. Pero resulta repetitivo que aquellos que cuentan con mayor cobertura de servicios, también son aquellos con mayores logros educativos (Ver Figura 7) 
Figura 7: Hogares que acceden al servicio de internet, comparativo 2008 Vs. 2018 por departamentos (Perú)

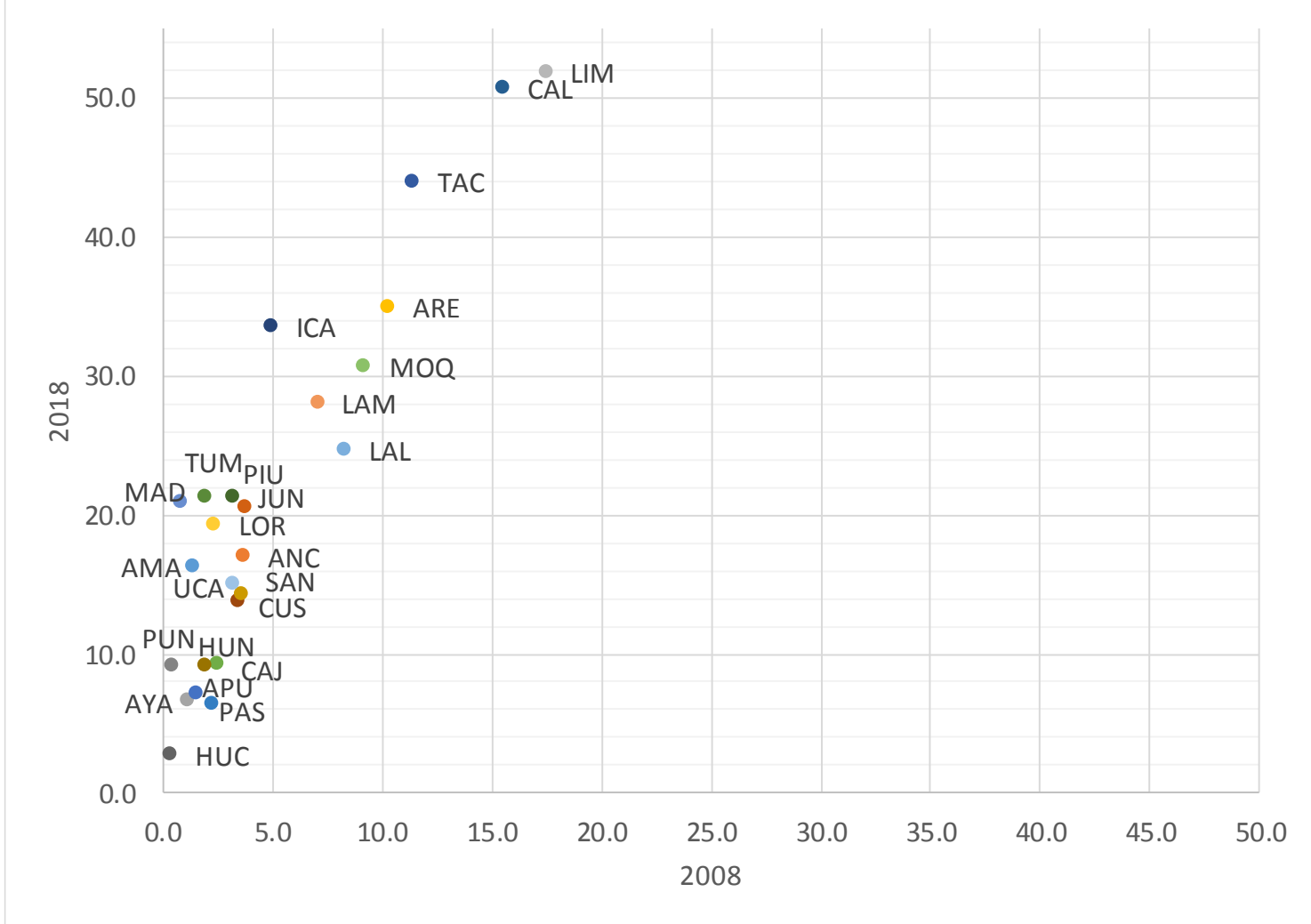

Fuente: Elaboración propia con información de (INEI, Instituto Nacional de Estadística e Informática-INEI, 2020)

Para determinar la relación entre la comprensión lectora y las demás variables independientes, se ha construido un modelo de sección cruzada, donde se ha analizado la evolución de cada variable en el periodo 2007-2016 para cada uno de los departamentos del Perú. El modelo busca determinar la relación que existe en el nivel de comprensión lectora: Alumnos que logran los aprendizajes del grado (\% de alumnos de $2^{\circ}$ grado participantes en evaluación censal) que alcanzan los departamentos que conforman el Perú y cómo influye sobre esta las variables analizadas siguientes:

- Porcentaje de locales escolares con aulas en buen estado. (estado_local_escolar)

- Gasto corriente percápita por alumno matriculado ejecutado por el Estado. (gasto_corriente_alumno)

- Desnutrición Crónica (desn)

- Porcentaje de hogares que se abastecen de agua mediante red pública. (agua) 
- Porcentaje de Hogares en viviendas particulares que disponen de alumbrado eléctrico por Red Pública. (luz)

- Porcentaje de Hogares que acceden al servicio de internet. (inter)

\section{ANÁLISIS DE RESULTADOS y DISCUSIONES}

El objetivo de la investigación es determinar el nivel de significancia de la inversión pública en educación como determinante de los logros educativos en el Perú. A partir de ello, la hipótesis de investigación plantea que la inversión pública ejecutada en educación no tiene un grado de influencia significativo en los logros educativos. De acuerdo al marco teórico revisado, se tiene que los logros educativos están determinados por los años de estudio, el set de características del colegio y los profesores, las características del niño, las características del hogar que afectan el aprendizaje y los insumos para la educación que el hogar aporta. Pero, para el caso en evaluación, se han analizado variables que estén relacionadas con la inversión pública, a fin de analizar aquellas que representen a los determinantes de los logros educativos mencionados anteriormente.

Al respecto, uno de los resultados de la presente investigación está referido al descarte de la variable inversión pública ejecutada como determinante de los logros educativos. Los resultados no han sido significativos para el caso del Perú, donde al analizar econométricamente la significancia de esta variable, en todos los modelos analizados ha reportado un nivel de significancia sobre el 5\%, que para efectos estadísticos no es un resultado concluyente. En ese sentido, este resultado no permite afirmar que la inversión pública en el periodo analizado haya tenido una influencia en los logros educativos. Este resultado contrasta con lo señalado por (Bayona Rodriguez, 2016), quien nos explica la importancia de la inversión en educación. Sin embargo, los hallazgos de la presente investigación indican que existen otras variables más relevantes, como son las condiciones que ofrecen los hogares para la educación, que influyen preponderantemente en los logros educativos. 
Dado que la variable de inversión pública en educación analizada no resultó concluyente, se analizó una variable proxy para ella. En este caso se utilizó para el modelo el porcentaje de instituciones educativas en buen estado. Al respecto, se tiene un modelo que relaciona las variables con un nivel de significancia menor al 5\%, como se exige para que una variable sea considerada como parte del modelo. Estos resultados nos permiten explicar los logros educativos, siendo concluyentes para afirmar que la desnutrición tiene una relación negativa con los logros educativos. En el caso de las instituciones educativas en buen estado, la cobertura de servicios de agua, luz e internet, se tiene una relación positiva con los logros educativos. Y finalmente, en el caso del gasto corriente per cápita por alumno, se presenta también una relación positiva. Al respecto, (Duarte \& Carlos \& Moreno, 2011) explican de sus análisis que la infraestructura con áreas complementarias en las escuelas tienen efectos sobre el aprendizaje, complementado por (Palacios, 2018) que analizan el agravamiento de las brechas de infraestructura educativa. Sin embargo, para el caso analizado, las variables que destacan están relacionadas a desnutrición y las condiciones de estudio que dan los hogares, como son agua, luz y acceso a internet.

A continuación, se presenta los resultados del modelo econométrico analizado, con el reporte de los valores de los coeficientes resultantes del modelo (Ver Tabla 2).

Tabla 2: Ecuación del Modelo Econométrico Utilizado

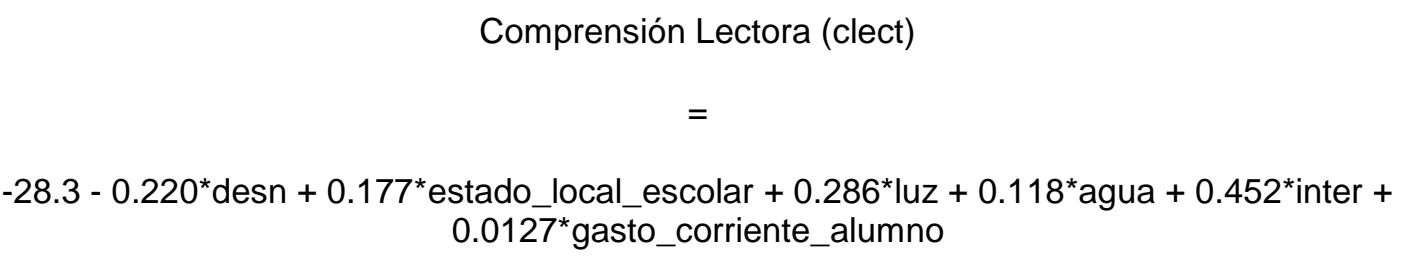

Fuente: Elaboración propia.

También, los resultados de los estadísticos resultantes para cada una de las variables se presentan seguidamente (Ver tabla 3). 
Tabla 3: Modelo: MC. Ponderados (utilizando 158 observaciones. Se han incluido 25 unidades de sección cruzada. Variable dependiente: clect. Ponderaciones basadas en varianzas de los errores por unidad)

\begin{tabular}{lllll}
\hline & Coeficiente & Desv. Típica & Estadístico $\boldsymbol{t}$ & valor $\boldsymbol{p}$ \\
\hline const & -28.2918 & 8.74588 & -3.235 & 0.0015 \\
desn & -0.220443 & 0.0757492 & -2.910 & 0.0042 \\
estado_local_escolar & 0.177494 & 0.0561666 & 3.160 & 0.0019 \\
luz & 0.285730 & 0.104124 & 2.744 & 0.0068 \\
agua & 0.117718 & 0.0515897 & 2.282 & 0.0239 \\
inter & 0.451881 & 0.100123 & 4.513 & $<0.0001$ \\
gasto_corriente_alumno & 0.0126836 & 0.00104707 & 12.11 & $<0.0001$ \\
\hline
\end{tabular}

Fuente: Elaboración propia utilizando software GRETL

Se observa que todas las variables independientes son significativas y no se deben excluir como variables explicativas del modelo, dado que presentan valores "p" con niveles de significación menores al 5\%. Asimismo, se tienen que el coeficiente de determinación (R-cuadrado), que estima la bondad de ajuste del modelo, estima que el $84.1 \%$ de las variaciones en la comprensión lectora son explicadas por las variables independientes analizadas. Esto se muestra a continuación.

\section{Tabla 4: Estadísticos del modelo}

\begin{tabular}{lcll}
\hline Suma de cuad. residuos & 151.2393 & D.T. de la regresión & 1.000792 \\
R-cuadrado & 0.841693 & R-cuadrado corregido & 0.835402 \\
$\mathrm{~F}(6,151)$ & 133.8069 & Valor $\mathrm{p}($ de F) & $7.70 \mathrm{e}-58$ \\
Log-verosimilitud & -220.7375 & Criterio de Akaike & 455.4750 \\
Criterio de Schwarz & 476.9131 & Crit. de Hannan-Quinn & 464.1813 \\
\hline \multicolumn{2}{l}{ Fuente: Elaboración propia utilizando software GRETL. }
\end{tabular}

De otro lado, se puede comparar los resultados de dos regiones. Primero el caso de Tacna, que es el departamento con el mejor desempeño en comprensión lectora. Y el caso de Loreto, que es el departamento con el menor desempeño en comprensión lectora. En el caso de Tacna, utilizando la ecuación estimada, se tiene que esta presenta para el 2016 un nivel de comprensión lectora del 76.8\%. En el caso de Loreto, este valor se sitúa en 17.7\% (Ver Tabla 5Tabla 5).

Tabla 5: Valores de las variables para Tacna y Loreto

\begin{tabular}{lrrr}
\hline Variables & Tacna & Loreto & Brecha \\
\hline \% Alumnos en Comprensión Lectora & 76.8 & 17.7 & 59.06 \\
\% de Desnutrición & 2.3 & 23.6 & -21.32 \\
\% I.E. en buen estado & 65.8 & 11.1 & 54.76 \\
\% Cobertura de Luz Eléctrica & 95.9 & 77.9 & 17.97 \\
\% Cobertura de agua potable & 94.3 & 55.3 & 39.00 \\
\% Cobertura Internet & 35.5 & 14.5 & 21.00 \\
Gasto Corriente Percápita Annual (En Soles) & $2,143.4$ & $2,026.3$ & 117.13 \\
\hline
\end{tabular}

Fuente: Elaboración propia. Datos de fuentes oficiales consultadas para la presente investigación. 
El modelo permite establecer comparaciones y explicaciones de por qué Tacna tiene un nivel más alto respecto a la Comprensión Lectora. Si se compara, en el caso de la desnutrición, Tacna presenta un nivel de $2.3 \%$, frente a Loreto que presenta un $23.6 \%$. Asimismo, en cuanto a Instituciones Educativas en buen estado, Tacna registra un $65.8 \%$, por el contrario, Loreto presenta un $11.1 \%$. De los anterior, se puede deducir que el cierre de brechas en cuanto a los logros educativos en el caso peruano tiene que ver con cerrar las diferentes brechas en cuanto a desnutrición y la cobertura de servicios básicos.

Sin embargo, otro tipo de análisis que permite realizar el presente modelo, es buscar responder a la pregunta de en cuanto se debe variar cada una de sus variables si se busca mejorar el desempeño de los alumnos en cuanto a comprensión lectora. Al respecto, si el objetivo de política educativa en el departamento de Loreto es incrementar el porcentaje de alumnos en comprensión lectora en un punto porcentual, esto se puede lograr reduciendo la desnutrición en 4.5 puntos porcentuales de su nivel actual. Pero también, se puede lograr el mismo efecto si se incrementa en 5.6 puntos porcentuales el porcentaje de aulas en buen estado. Del mismo modo, se puede lograr el mismo incremento si se aumenta la cobertura de luz eléctrica en 3.5 puntos porcentuales, o en 8.5 puntos porcentuales en la cobertura de agua potable o en 2.2 puntos porcentuales la cobertura de internet. También, si se incrementa en 78.8 soles el gasto corriente per cápita por alumno en un año se tendrá un incremento de un punto porcentual en la comprensión lectora (Ver Tabla 6).

Tabla 6: Estimación de la variación necesaria para incrementar la comprensión lectora en $1 \%$ en las variables que la determinan.

\begin{tabular}{lcc}
\hline Variables & $\begin{array}{c}\text { Variación en puntos } \\
\text { porcentuales de su } \\
\text { valor inicial }\end{array}$ & $\begin{array}{c}\text { Variación en \% que } \\
\text { representa el cambio } \\
\text { de puntos } \\
\text { porcentuales }\end{array}$ \\
\hline \% Alumnos en Comprensión Lectora & 1.0 & $3.4 \%$ \\
\% de Desnutrición & -4.5 & $-19.2 \%$ \\
$\%$ I.E. en buen estado & 5.6 & $51.0 \%$ \\
\% Cobertura de Luz Eléctrica & 3.5 & $4.5 \%$ \\
\% Cobertura de agua potable & 8.5 & $15.4 \%$ \\
$\%$ Cobertura Internet & 2.2 & $15.3 \%$ \\
Gasto Corriente Per cápita Anual (En & 78.8 & $3.9 \%$ \\
Soles) & &
\end{tabular}

Fuente: Elaboración propia. 


\section{CONCLUSIONES}

Los resultados anteriores permiten plantear lo siguiente: Respecto a la variable inversión pública per cápita por alumno en educación, se ha hallado que no tiene una asociación significativa econométricamente entre la inversión pública en educación y la comprensión lectora. Pero, cuando se analiza la inversión pública como generadora de instituciones educativas en buen estado, se encuentra que esta variable si es significativa como variable explicativa de los logros educativos. Sin embargo, no resulta adecuado atribuir enteramente la disponibilidad de instituciones educativas en buen estado a la inversión pública, dado que esta condición se puede perder si no hay un adecuado mantenimiento de la infraestructura existente, que corresponde más bien a una porción del gasto corriente. También, para el gasto corriente per cápita por alumno, este ha demostrado para todos los modelos analizados su significancia como variable explicativa de los logros educativos. Además, en cuanto a la desnutrición, esta ha demostrado ser significativa en todos los niveles. Esta se relaciona con garantizar que los estudiantes tengan como característica intrínseca la plena disponibilidad de sus capacidades. En el caso de las variables de cobertura de servicios de agua, luz e internet, estas han demostrado su importancia como parte del grupo de factores necesarios con los que contribuye el hogar para que los alumnos puedan estudiar. Entonces, los logros educativos se ven influenciadas no solo de variables relacionadas únicamente con la educación, sino también con variables de la salud, la economía o el acceso a información. Por tanto, estas variables se constituyen, a partir de esta investigación, en variables relevantes a considerar en cualquier política de mejora de los logros educativos. Asimismo, revelan el carácter multisectorial que deben tener las políticas educativas. 


\section{REFERENCIAS BIBLIOGRÁFICAS}

Allanta, A. (2017). Infraestructura Básica Regular para lograr una Educación de calidad en el distrito La Yarada, Los Palos, Tacna. [Tesis de Maestría, Universidad Privada de Tacna]

Anahua, E. (2017). La gestión educativa y el compromiso laboral en la dirección regional sectorial de educación de Tacna. [Tesis de Maestría, Universidad Privada de Tacna]

Arce Vega, P. J., \& Tejada Vidal, N. G. (2017). Impacto de la inversión pública en el crecimiento de la Región Cajamarca - período 2008-2017. REVISTA GOBIERNO Y GESTIÓN PÚBLICA, $\quad 4(1), \quad 117 \quad$ - 138. doi:https://doi.org/10.24265/iggp.2017.v4n1.07

Bayona Rodriguez, H. (2016). Efectos de la infraestructura sobre el fracaso esclar: evidencia empírica para Colombia. [Tesis de Maestría, Universidad de los Andes] doi:http://dx.doi.org/10.18175/VyS7.2.2016.03

Carnoy, M. (2013). Economía de la educación. Barcelona, España: Editorial UOC.

Carnoy, M., Miller, L., \& Luschei, T. (2006). Economía de la Educación. Barcelona, España: El Ciervo 96 S.A.

Consulta Amigable MEF, M. d. (07 de Junio de 2020). Transparencia Económica. $\begin{array}{llll}\text { Consulta de } & \text { Ejecución }\end{array}$ https://apps5.mineco.gob.pe/transparencia/Navegador/default.aspx?y=2019\&a $\mathrm{p}=$ Proyecto

Coraggio, J. L. (1995). Las propuestas del Banco Mundial para la educación. ¿sentido oculto o problemas de concepción? Argentina.

Cordero Ferrera, J. M., Crespo Cebada, E., \& Chaparro, P. (2011). Exploring educational efficiency divergences across spanish regions in PISA. Revista de Economía Aplicada, $X I X(57)$

pp. 117-145. https://www.redalyc.org/pdf/969/96922243005.pdf 
Duarte, J. \&., \& Carlos \& Moreno, M. (2011). Infraestructura escolar y aprendizajes en la educación básica latinoamericana: Un análisis a partir del SERCE.

ESCALE-Ministerio de Educación. (14 de Junio de 2020). ESCALE. Estadística de la Calidad Educativa. MINEDU-Ministerio de Educación: http://escale.minedu.gob.pe/indicadores

Fermoso, P. (1997). Manual de economía de la educación. Madrid, España: Narcea S.A. Glewwe, P., Lambert, S., \& Chen, Q. (2020). Chapter 15-Education production funcions: updated evidence from developing countries. En S. Bradley, \& G. Colin, The Economics of Education a comprehensive overview (Second Edition). London: Academic Press.

Hanushek, E. A. (2020). Chapter 13 - Education production functions. En S. Bradley, \& G. Colin, The Economics of Education a comprehensive overview (Second Edition) (págs. 161-170). London, United Kingdom: Academic Press. doi:https://doi.org/10.1016/B978-0-12-815391-8.00013-6.

Instituto Nacional de Estadística e Informática-INEI. (2019). Evolución de la Pobreza Monetaria 2007-2018. Lima: INEI.

Instituto Nacional de Estadística e Informática-INEI. (11 de Junio de 2020). Instituto Nacional de Estadística e https://www.inei.gob.pe/estadisticas/indice-tematico/education/

Instituto Nacional de Estadística e Informática-INEI. (15 de Junio de 2020). Sistema de Información Regional para la toma de decisiones. ENAHO: http://webinei.inei.gob.pe:8080/SIRTOD/inicio.html\#app=8d5c\&d4a2selectedIndex $=0 \& d 9$ ef-selectedIndex $=1$

JUNTOS. (05 de Mayo de 2020). JUNTOS. https://www.juntos.gob.pe/nosotros/quehacemos/

Lassibille, G. N. (Junio de 2012). Un compendio de investigación en economía de la educación. Presupuesto y Gasto Público, Institut de Recherche sur l'Economie de l'Education y Centre National de la Recherche Scientifique. Dijon. (Francia) 
Mg. Ronald Nilton Silva Gil. ORCID: 0000-0001-7221-6108

Dra. Nora Gina del Pilar Tejada Vidal. ORCID: 0000-0002-8498-9826

Mamani, D. (2018). Factores que influyeron en los resultados de lectura de la ECE 2016, en $2^{\circ}$ grado de educación secundaria en las instituciones educativas del cercado de Tacna. Tacna: Universidad Nacional Jorge Basadre Grohmann.

Ministerio de Economía y Finanzas-MEF. (11 de Junio de 2020). Ministerio de Economía y Finanzas. Consulta Amigable SIAF: https://apps5.mineco.gob.pe/transparencia/Navegador/default.aspx

Ministerio de Educación-MINEDU. (2016). El impulso de una carrera, política de revalorización docente en el Perú. Lima: Ministerio de Educación del Perú.

Ministerio de Salud-MINSA. (15 de Junio de 2020). REUNIS - Repositorio Único Nacional de Información en Salud. http://www.minsa.gob.pe/reunis/recursos_salud/index_desnutricion_cronica.asp Morduchowics, A. (2004). Discusiones de economía de la educación. Buenos Aires, $\begin{array}{lll}\text { Argentina: } & \text { Editorial }\end{array}$ https://unesdoc.unesco.org/ark:/48223/pf0000150777

Palacios, J. (2018). La inversión pública en educación y la brecha de infraestructura física en la educación básica regular durante el periodo 2000-2015. [Tesis de Maestría, Universidad San Martín de Porras]

Pilco, A. (2019). Plan lector en el logro de aprendizajes en los alumnos de secto grado de educación primaria en la institución educativa Hermanos Barreto de la ciudad de Tacna 2017. [Tesis de Maestría, Universidad Privada de Tacna]

Rodríguez Mendoza, E. (2018). Clima escolar y calidad educativa en la Institución Educativa Argentina-Lima, 2018. REVISTA GOBIERNO Y GESTIÓN PÚBLICA, 5(1), 51 - 72. doi:https://doi.org/10.24265/iggp.2018.v5n1.04

Salas Velasco, M. (02 de Agosto de 2001). Cuatro décadas en economía de la educación. Revista de Educación, 427-449. http://www.educacionyfp.gob.es/dam/jcr:399c556a-58ea-467e-861df27e6fa96f1d/re3282310861-pdf.pdf ISSN: 2414-4991. Creative Commons Atribución-NoComercial-Compartirlgual 4.0 Internacional. (c)(1)(2) 
Santusa, M. (2018). Influencia del Bullying en el aprendizaje de los estudiantes del segundo y cuarto año de educación secundaria en la institución educativa Carlos Armando Laura de Tacna. [Tesis de Maestría, Universidad Nacional Jorge Basadre Grohmann]

Scott, A. (2012). Northern Lights, The positive policy example of Sweden, Finland, Denmark and Norway. Australia: Monash University.

Organización de las Naciones Unidas para la Educación, la Ciencia y la CulturaUNESCO. (2017). Revisión de las políticas públicas del sector educación en Perú. . Francia: Organización de las Naciones Unidas para la Educación.

Zegarra, E., \& Ravina, R. (2003). Teacher Unionization and the Quality of Education in Peru: An Empirical Evaluation Using Survey Data. Washigton: Research Network Working papers; R-474. 